\title{
A correlative comparison of the ring current and auroral electrojets using geomagnetic indices
}

\author{
W. B. Cade III, ${ }^{1}$ J. J. Sojka, and L. Zhu \\ Center for Atmospheric and Space Science, Utah State University, Logan
}

\begin{abstract}
From a study of the 21 largest geomagnetic storms during solar cycle 21, a strong correlation is established between the ring current index Dst and the time-weighted accumulation of the 1-hour auroral electrojet indices, $A E$ and $A L$. The time-weighted accumulation corresponds to convolution of the auroral electrojet indices with an exponential weighting function with an $e$-folding time of 9.4 hours. The weighted indices $A E_{w}$ and $A L_{w}$ have correlation coefficients against Dst ranging between 0.8 and 0.95 for 20 of the 21 storms. Correlation over the entire solar cycle 21 database is also strong but not as strong as for an individual storm. A set of simple Dst prediction functions provide a first approximation of the inferred dependence, but the specific functional relationship of Dst $\left(A E_{w}\right)$ or Dst $\left(A L_{w}\right)$ varies from one storm to the next in a systematic way. This variation reveals a missing parametric dependence in the transfer function. However, our results indicate that auroral electrojet indices are potentially useful for predicting storm time enhancements of ring current intensity with a few hours lead time.
\end{abstract}

\section{Introduction}

In this work we search for a statistical relationship between auroral electrojet indices and the ring current index Dst. The existence of such a relationship seems plausible, since both phenomena are driven by the same basic energy source (the solar wind). A statistical association between the responsible current systems might clarify the relationship between storms and substorms, as well as aid in ring current modeling and/or prediction. Very few previous investigators, however, have looked for such a relationship.

The ring current intensity is monitored by the $D s t$ geomagnetic index. The auroral electrojet is monitored by indices called $A E, A L, A U$, and $A O$. The $A$ indices are proxy indices in that they do not quantify the actual currents directly. We use all these indices in the present correlative study.

One of the first direct comparisons between Dst and $A E$ was made by Davis and Parthasarathy [1967]. They found that (1) the onset of $A E$ activity precedes $D R$ (or $D s t$ ) enhancement by as much as 15 hours in geomagnetic storms; (2) the amplitude of the maximum $D R$ is directly proportional to the sum of the 1-hour $A E$ values for the previous 10 hours (they found a correlation coefficient of 0.82 between $D R$ and the 10-hour sum of $A E$ for 32 magnetic storms in 1958); and (3) the energy injection function to the ring current (derived by them) looks very similar to the time variation of $A E$, suggesting that both are energized by the same process.

Akasofu [1981b] made a direct comparison of the $A E$ and Dst indices for several different solar rotation periods. He

\footnotetext{
${ }^{\mathrm{l} N o w}$ at Air Force Space Command, Peterson Air Force Base, Colorado.
}

Copyright 1995 by the American Geophysical Union.

Paper number 94JA 02347.

0148-0227/95/94JA-02347\$05.00 concluded that, while there is a linear relationship at relatively quiet periods (Dst $>-50 \mathrm{nT}$ ), there is no longer such a relationship during storms because the storm time $A E$ index has rapid fluctuations that enable it to be relatively high or relatively low. Hence there was a wide spread in the scatterplot of Dst versus $A E$.

Several methods for modeling Dst (and hence the ring current) have been developed over the past 30 years. An early effort by Kamide and Fukushima [1971] attempted to use $A E$ times an exponential function of time to model the energy input into the symmetric ring current. By varying the decay time in the equation for energy change of the ring current, they were able to represent $D s t$ fairly well. However, this was done for only one storm, and no further work in this area was pursued.

Akasofu [1981a] used his $\varepsilon$ parameter to model $A E$ and Dst to show that both are directly driven by the solar wind. $\mathrm{He}$ achieved good agreement for a few storms and indicated the possibility of forecasting these indices from interplanetary magnetic field (IMF) measurements.

Similarly, Siscoe [1982] proposed that the $A E$ index varies linearly with the $B_{z}$ component of IMF while Dst varies as $B_{z}^{2}$. Both these relationships are directly dependent upon the solar wind, in contrast to the work of Davis and Parthasarathy [1967] and Kamide and Fukushima [1971] which indicate that $D s t$ depends upon the past history of $A E$ rather than upon its present value.

Feldstein [1992] reviewed various models that use IMF parameters to predict Dst. He tested several of them and determined that the method developed by Pisarsky et al. [1989] was one of the better. One difficulty with the method of Pisarsky et al., however, is that it requires prior knowledge of the peak Dst and so cannot model Dst in real time. This method suggests that the IMF to Dst transfer function is nonlinear and depends on Dst itself. Other methods, such as that introduced by Burton et al. [1975], could model Dst in real time if the IMF were always available. However, IMF data are 
not always available during a storm, much less so without a time delay.

In this study we use the hourly auroral $A$ indices as a measure of auroral currents. We gather from the work of Fairfield [1992] that the hourly $A E$ corresponds to directly driven processes. The work of Cade [1993] corroborates this interpretation. The first objective of the present work is to determine whether the Dst index is directly driven or instead more accurately characterized as responsive to the recent history of directly driven activity. Our second objective is to determine what functional representation might relate $D s t$ to the $A$ indices. The database for our study comprises solar cycle 21. We define a mathematical procedure for deriving Dst from the past history of the hourly auroral indices and apply it to the 21 largest storms during solar cycle 21 , as well as to the entire 11-year period. We discuss contrasts between our results and earlier work enabling us to better understand how the ring current is generated.

\section{Indices Used in This Study and Their Limitations}

The Dst index was first derived both by Sugiura [1964] and Kertz [1964]. It was conceived as a measure of the intensity of the equatorial ring current, but the effects of the partial ring current and of magnetospheric compression by the solar wind are not removed, and some residual effects may remain from regular variations even though procedures are used to remove them [Mayaud, 1980]. This and the other indices that constitute our database were obtained from the coupling energetics and dynamics of atmospheric regions (CEDAR) database at the National Center for Atmospheric Research (NCAR).

The auroral $A$ indices $(A E, A L, A U$, and $A O$ ) were first introduced by Davis and Sugiura [1966] to measure the auroral electrojets and were discussed in detail by Mayaud [1980]. AL should measure the intensity of the westward electrojet, whereas $A U$ should measure the intensity of the eastward electrojet. However, these indices also contain contributions from any other zonal currents (in the ionosphere and magnetosphere), mainly the ring current, thus $A E$ is defined as $A U-A L$ so as to remove any symmetric zonal contribution. $A O$, defined as $(A U+A L) / 2$, is then intended to be an approximate measure of the equivalent zonal current. Since there is asymmetry between the eastward and westward electrojets, however, $A O$ really measures this asymmetry plus zonal current effects.

As is noted above, Akasofu [1981b] had found a linear correlation between $A E$ and $D s t$ under nonstorm conditions but none during storms. In view of our interest in strongly disturbed conditions, we focus our attention on storm periods. Storms can be defined in many ways. They can be classified in terms of the geomagnetic indices $A p, a p, K p$, or $D s t$, or in terms of effects, either terrestrial or orbital. For the present study we have adopted but modified the criterion used by NOAA's Space Environmental Services Center. Their criterion for a severe geomagnetic storm is that the $A p$ be $\geq 100$ (W. Cliffswallow, private communication, 1992). We require either $A p^{*} \geq 100$ ( $A p$ is monitored from 0000 to $2400 \mathrm{UT}$, while $A p^{*}$ is the maximum value of $A p$ centered on a 24-hour interval) or that Dst $\leq-200 \mathrm{nT}$ (to be sure that no storms with large ring current intensification were missed).

We applied these criteria to the database of geomagnetic indices from 1978 to 1986 (solar cycle 21). Table 1 shows data for the 21 storms that met either or both of the above criteria, and Figure 1 shows the superposed Dst traces for the 21 storms. It is a superposed epoch representation showing Dst for each storm from 48 hours before until 72 hours after the most negative value of $D s t$ was attained (at the time we call $t=$ 0 ). This superposition reveals a similarity in the profiles for most of the storms (except for a couple of storms which have

Table 1. Summary of Conditions for the 21 Storms Selected From Solar Cycle 21

\begin{tabular}{|c|c|c|c|c|c|}
\hline $\begin{array}{l}\text { Storm } \\
\text { Number }\end{array}$ & Date & $\begin{array}{c}\text { 81-Day } \\
\text { F10.7 }\end{array}$ & $A p^{*}$ & $\begin{array}{c}\text { Minimum } \\
\text { Dst }\end{array}$ & $\begin{array}{l}\text { Maximum } \\
\text { 3-hour } K_{p}\end{array}$ \\
\hline 1 & May $1-4,1978$ & 142 & 130 & -150 & $8+$ \\
\hline 2 & Aug. 27-30, 1978 & 135 & 128 & -226 & $8+$ \\
\hline 3 & Sept. $27-30,1978$ & 152 & 122 & -224 & 80 \\
\hline 4 & April 3-6, 1979 & 178 & 91 & -202 & 80 \\
\hline 5 & April 24-28, 1979 & 177 & 126 & -149 & $8 \circ$ \\
\hline 6 & Dec. $19-22,1980$ & 193 & 80 & -240 & $8-$ \\
\hline 7 & March 5-8, 1981 & 209 & 82 & -215 & $7+$ \\
\hline 8 & April 11-14, 1981 & 212 & 134 & -311 & $8+$ \\
\hline 9 & July $24-27,1981$ & 206 & 161 & -226 & $8+$ \\
\hline 10 & March 1-4, 1982 & 202 & 140 & -211 & 80 \\
\hline 11 & July $13-16,1982$ & 172 & 230 & -325 & 90 \\
\hline 12 & Aug., 6-9, 1982 & 166 & 116 & -155 & $8-$ \\
\hline 13 & Sept. 5-8, 1982 & 163 & 201 & -289 & 9- \\
\hline 14 & Sept. $21-24,1982$ & 163 & 148 & -210 & $8+$ \\
\hline 15 & Jan. 9-12, 1983 & 155 & 86 & -213 & $8+$ \\
\hline 16 & Feb. 4-8, 1983 & 128 & 158 & -183 & 80 \\
\hline 17 & April 25-29, 1984 & 128 & 103 & -93 & $8-$ \\
\hline 18 & Sept. 22-25, 1984 & 77 & 115 & -75 & 7。 \\
\hline 19 & Nov. 15-18, 1984 & 73 & 125 & -141 & 80 \\
\hline 20 & April 19-23, 1985 & 78 & 118 & -158 & $8+$ \\
\hline 21 & Feb. $7-10,1986$ & 79 & 229 & -307 & 90 \\
\hline
\end{tabular}

$A p^{*}$ is the maximum value of $A p$ centered on a 24-hour interval. 


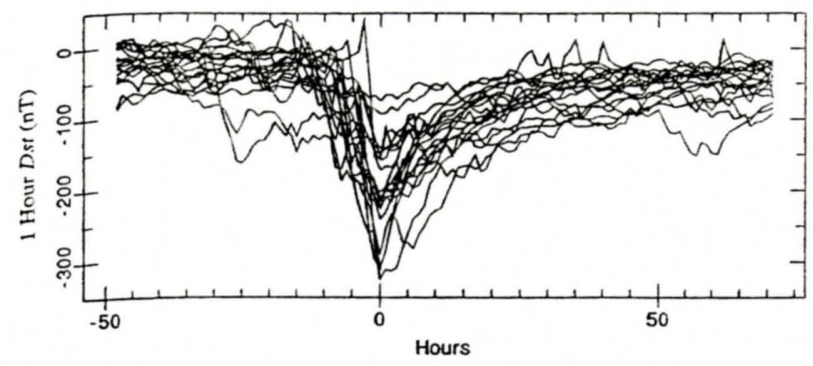

Figure 1. Superposed Dst traces for the 21 storms from solar cycle 21 . The minimum (most negative) Dst of each trace defines $t=0$.

significantly more negative $D s t$ as prestorm conditions), a main phase rise of about 5 to 10 hours, and a decay (recovery phase) lasting about 2 days.

\section{Analytical Procedure}

In this work we demonstrate a delayed correlation between Dst and a cumulative version of the $A$ indices. Our rationale for this approach is historically well founded: Akasofu [1981b] showed that $A E$ and $D s t$ are linearly related for only weakly negative values of $D s t, \gtrsim-50 \mathrm{nT}$. For more strongly negative values the relationship is more complicated. Davis and Parthasarathy [1967] found that summing the prior 10 hours of $A E$ produced an index which varied somewhat like $D s t$, and Kamide and Fukushima [1971] used an exponentially weighted integration over prior $A E$ values to obtain a very good correlation with Dst during one major storm. Wrenn [1989] compared the 3-hour Ap to Dst by using a weighted summation of earlier $A p$ values. His technique is particularly straightforward and associates a time constant with the weighting function. This weighting scheme and the associated time constant are defined in the appendix. It is a discrete summation method equivalent to the integral scheme used by Kamide and Fukushima. Thus for a given time series of hourly $A$ indices, an equivalent time series of the new weighted sum index $A_{w}$ is computed. The degree of weighting is specified by the parameter $w$, as a weight $w^{n}$ is applied to the $n$th hourly interval prior to the time of interest. Figure 2 shows the $A L$ index for December 19-22, 1980, and illustrates the effect of two different values of $w$ on the new index. As $w$ is increased from 0.6 to 0.9 , the $A L_{w}$ index becomes more dependent upon the past history of the $A L$ index. The minimum in the $A L$ index occurred on December 19, 1980, at 1400 UT, whereas the minima for $A L_{w}$ with $w=0.6$ and 0.9 occur later, at 1700 and 2000 UT, respectively. The Dst minimum (most negative value) for this period (bottom panel, Figure 2) occurred at 1900 UT on the same day. A progressively better correlation is obtained by comparing $A L, A L_{\mathrm{w} 0.6}$, and $A L_{\mathrm{w} 0.9}$ with $D s t$. Thus we performed a simple regression analysis to find the value of $w$ that gave the best correlation between $A L_{w}$ and $D s t$. We did the same thing for the $A E_{w}$ index.

\section{Comparison of $D s t$ to $A E_{w}$ and $A L_{w}$ for Storms}

For each storm we found the best value of $w$ for correlating each $A_{w}$ index with $D s t$ as in Figure 2 [cf. Cade, 1993]. Each
index had a

index had a slightly different optimal value of $w$, which varied
slightly from one storm to the next. However the best $w$ values clustered around $w=0.9$, which is equivalent to a time constant of $\tau=9.5$ hours. For the $A L_{w}$ and $A E_{w}$ indices the spread about $\tau=9.5$ hours was about \pm 2 hours. Storm 3, September 27-30, 1978, was an exception to this. From Figure 2 it is clear that the 1-hour $A L$ reached its most negative value several hours before $D s t$ did, but that the $A L_{w}$ index becomes more synchronized with $D s t$ as $w$ is increased from 0.6 to 0.9 . The following analysis was performed to verify that this time shift is statistically meaningful: $A E$ (Figure 3) and $A L$ (Figure 4) were time shifted relative to $D s t$ to produce optimal correlation coefficients for each storm. (The thin lines in Figures $3 \mathrm{a}$ and $4 \mathrm{a}$ show the absolute correlation coefficients for $D s t$ with the $A E$ and $A L$ indices, while the top panels of Figures $3 \mathrm{~b}$ and $4 \mathrm{~b}$ show the time shift needed to get this maximum correlation.) Adopting $w=0.9$ for all storms and indices, Figures 3 and 4 show the equivalent time shift analysis for $A E_{w}$ and $A L_{w}$. Two things are of note: the maximum correlation coefficients for the $A_{w}$ indices are dramatically better than for the $A$ indices, and the time shifts for the $A_{w}$ indices are centered about 0 hours. This latter finding is reassuring in that it demonstrates that the weighted sum of the past history of the $A$ indices is introducing a physically meaningful delay. It is consistent with the idea that the Dst responds to the recent past history of geomagnetic activity, as indicated by $A L$ and $A E$.

The results for $A O$ (not shown) were somewhat better than for $A L$. For $A O_{w}$ the correlation coefficients ranged from 0.829 to 0.955 (except for storm 1 which was 0.744 ), with 10 storms above 0.90 . Lead times for $A O$ were centered on $\sim 1$ to 2 hours, while lead times for $A O_{w}$ were 0 or negative. However, $A O_{w}$ is an index heavily influenced by the ring current, and so a

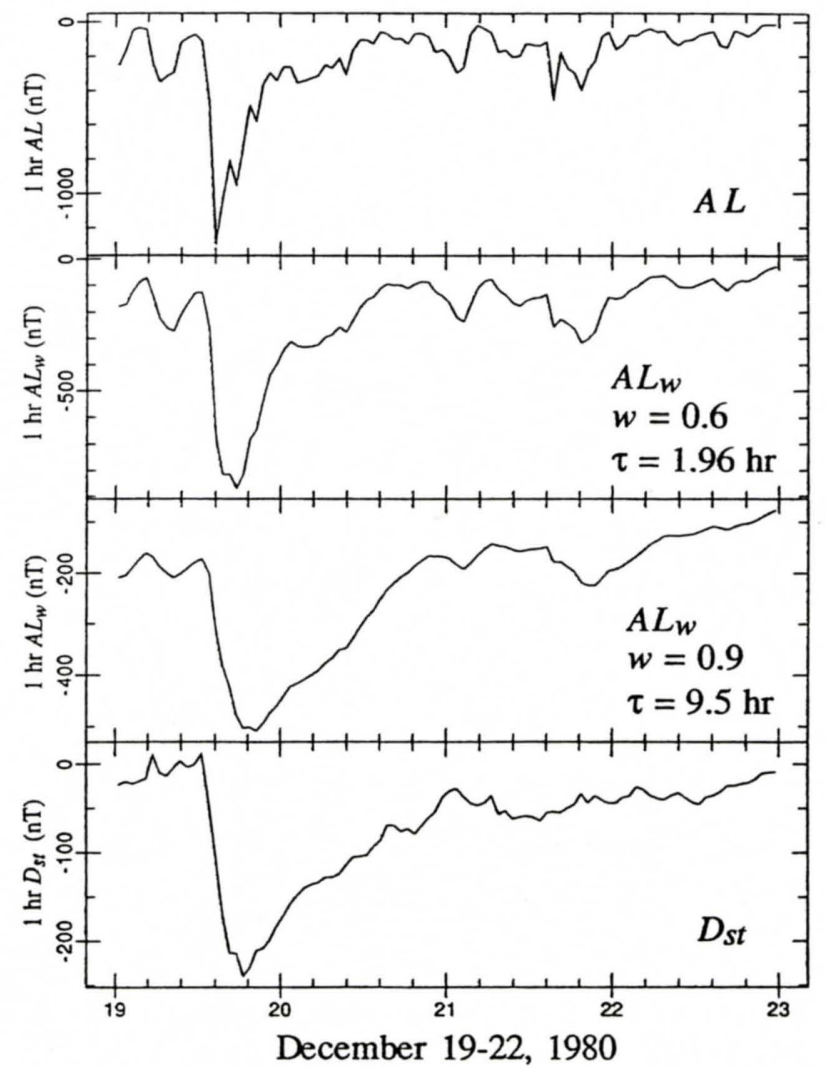

Figure 2. $A L$ and $A L_{w}$ for $w=0.6$ and 0.9 and $D s t$ for December 19-22, 1980. 


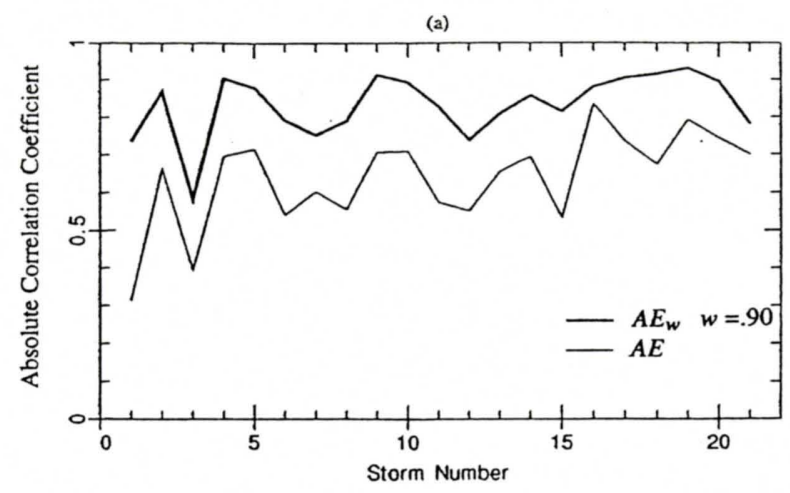

(b)

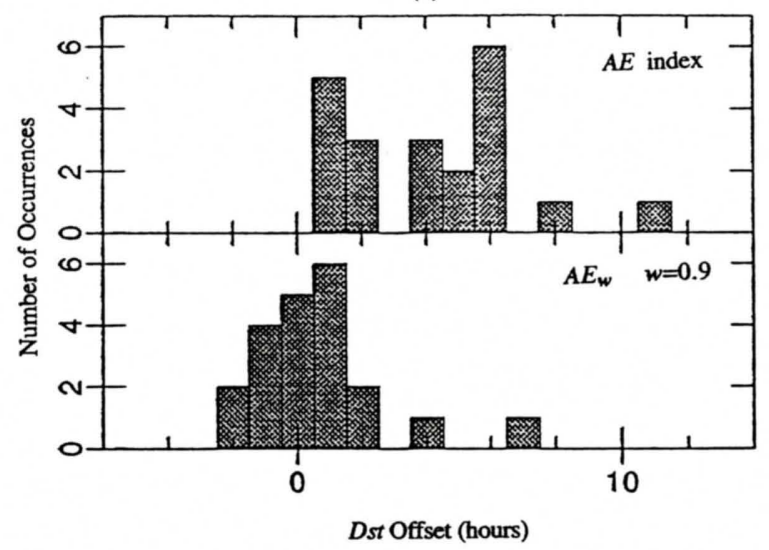

Figure 3. (a) Correlation coefficients and (b) time shifts needed to achieve maximum correlation for $A E$ and $A E_{w}$ compared to Dst.

good correlation for it has less physical significance than for $A E$ and $A L$. Therefore $A O$ is not considered further in this study. The results for $A U$ were less consistent and significantly poorer than for the other indices. The correlation coefficients for $A U_{w}$ ranged from -0.574 to -0.919 , with the maximum (most negative) correlation for the straight index often shifted by 24 hours (the maximum allowed in this study).

\section{Annual Prediction of $D s t$ From $A E_{w}$ and $A L_{w}$}

Given the high correlations found between the $A_{w}$ indices and $D s t$ for the large storms, it is possible that $A E_{w}$ and $A L_{w}$ could be used to model and forecast $D s t$ under all conditions. The year 1982 was chosen for use in determining the relationship between these indices and Dst, since this year contained the largest number of intense storms ( 5 of the 21 ) in the database. $A E_{w}$ and $A L_{w}$ were then calculated with $w=0.9$ for the entire year and compared to Dst. The correlation coefficients for the year were -0.751 for $A E_{w}$ and 0.792 for $A L_{w}$, compared to -0.571 for $A E$ and 0.584 for $A L$. Figure 5 shows a scatterplot of $A E_{w}$ versus $D s t$ for the entire 1982 data set. It is apparent in the plot that a reasonably linear relationship exists for lower values of $A E_{w}$ up to arrow $\mathrm{A}$, whereas a different relationship holds at higher values. The position of arrow A on Figure 5 is given by the intersection of the linear Dst fit (equation (1a): see below) with the function fitting the more negative $D s t$ values (equation (2a)). At the intersection the Dst has a value of $-64 \mathrm{nT}$. This value

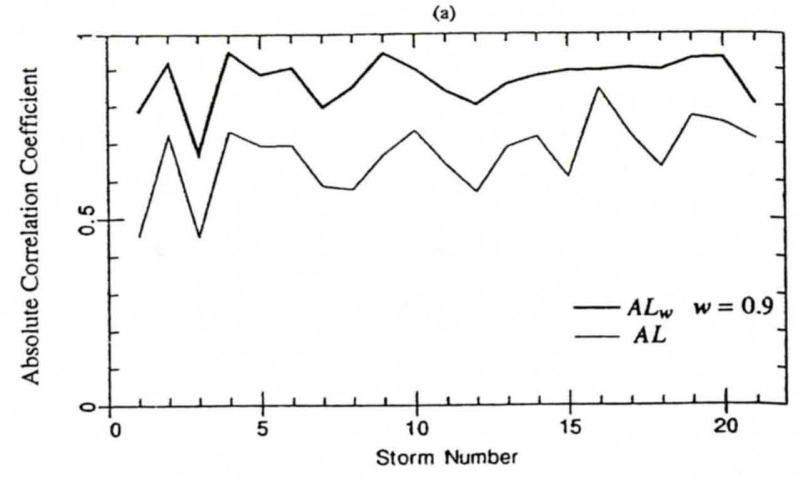

(b)

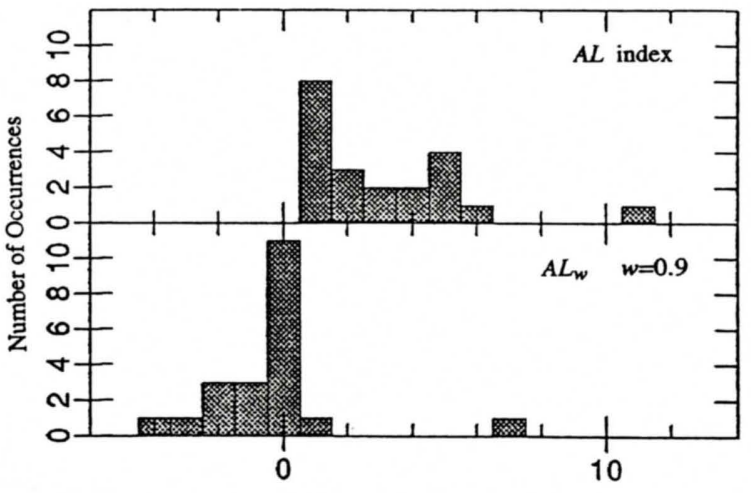

Dst Offset (hours)

Figure 4. (a) Correlation coefficients and (b) time shifts needed to achieve maximum correlation for $A L$ and $A L_{w}$ compared to Dst.

corresponds well with the result of Akasofu [1981b], who

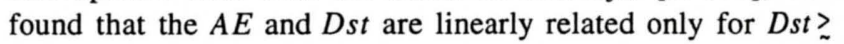
$-50 \mathrm{nT}$.

To determine the relationship of the linear portion, a least squares fit was performed on the 1982 data set (the resulting line for $A E_{w}$ is shown in Figure 5). The resulting equations for $A E_{w}$ and $A L_{w}$ were

$$
\begin{aligned}
& \operatorname{Dst}\left(A E_{w}\right)=\left[-0.147 \times A E_{w}\right]+20.2 \mathrm{nT} \\
& \operatorname{Dst}\left(A L_{w}\right)=\left[0.224 \times A L_{w}\right]+17.2 \mathrm{nT}
\end{aligned}
$$

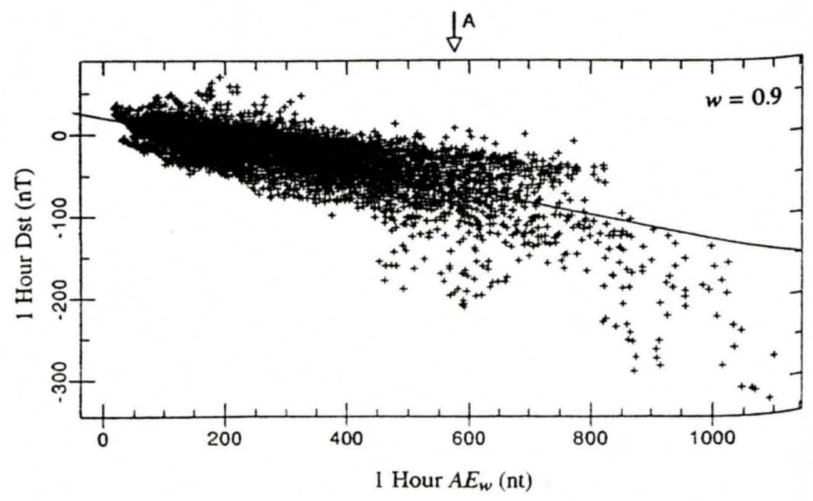

Figure 5. Plot of $D s t$ versus $A E_{w}$ for 1982. The linear fit was determined from a least squares method. Arrow A is the point below which a linear relationship holds. 
For values above arrow A, an exponential fit was found to work best, even though for each individual storm a different exponential or even a linear fit may hold. This issue will be further explored in the next section. Values of Dst, $A E_{w}$, and $A L_{w}$ were taken for 65 deepest $D s t$ minima during the year and fitted with a least squares fit to produce the following equations for $A E_{w}$ and $A L_{w}$ :

$$
\begin{aligned}
& \operatorname{Dst}\left(A E_{w}\right)=-13.2 \times \exp \left(0.0017 A E_{w}\right) \mathrm{nT} \\
& \operatorname{Dst}\left(A L_{w}\right)=-13.8 \times \exp \left(-0.004 A L_{w}\right) \mathrm{nT}
\end{aligned}
$$

The 65 Dst values used for this procedure and the least squares fit for $A E_{w}$ are shown in Figure 6.

The fits represented by (1) and (2) intersect. The intersection points define the $A E_{w}$ and $A L_{w}$ values at which the computation of Dst transfers from (1) to (2). For $A E_{w}$ the intersection is at $A E_{w}=575 \mathrm{nT}$ and corresponds to Dst $=-64$ nT. For $A L_{w}$ the intersection is at $A L_{w}=-450 \mathrm{nT}$ and corresponds to Dst $=-83 \mathrm{nT}$. Equations (1) and (2) were then used to predict Dst for 1982 (see Figure 7). The correlation coefficient is 0.775 for $A E_{w}$ and 0.793 for $A L_{w}$. These correlation coefficients are not quite as good as those for the entire ensemble of 21 storms (Figures $3 a$ and $4 a$ ), for which the values were mostly $>0.8$. Further discussion of this point is given in the next section.

Since (1) and (2) were developed from the 1982 data, one would expect a reasonable correlation when compared with 1982 data. To test the derived relations for a more general validity, they were applied to the other years covered in this study (1978-1986), and the results are given in Table 2. For most years the relationships specified by (1) and (2) worked just as well as for 1982; for some years (1981 and 1984) they were even superior, yielding correlations of 0.8 or better. As an example of application to another year, Figure 8 shows Dst compared to Dst $\left(A E_{w}\right)$ for 1978.

In both Figures 7 and 8 there are times when the agreement breaks down. This can happen during storm times but also during quiet periods. For example, the interval from day 194 through day 198 in 1978 (Figure 8) is a quiet period (prestorm), but yet the prediction for Dst is off by $\sim 30 \mathrm{nT}$ throughout. By contrast, the difference is $\lesssim 1 \mathrm{nT}$ in the poststorm interval from day 203 to day 213 in 1978 . For the case of storms it is relatively easy to find discrepancies as well as examples of reasonably good agreement. The storms on day 186, 1982 (Figure 7) and on days 195 and 265 in 1978 (Figure 8) show very good agreement, while the predictor

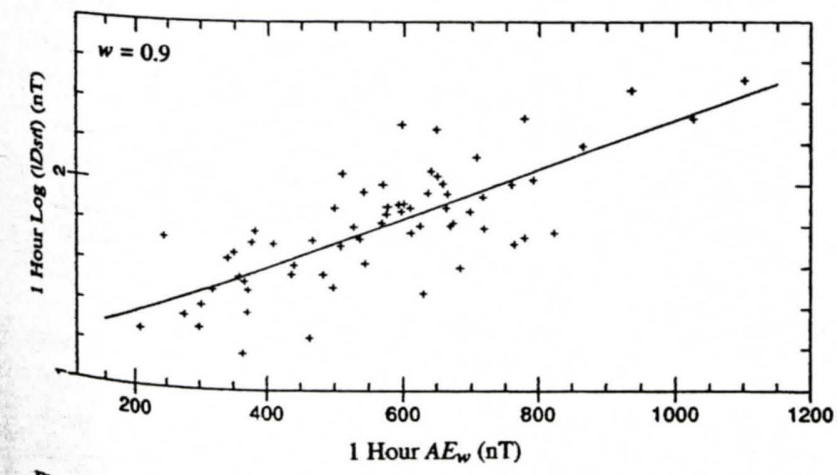

Pigure 6. Plot of 65 deepest Dst minima in 1982 versus $A E_{w}$. The linear fit was determined from a least squares method. underestimates the observed Dst by a factor of 2 on day 240 , 1982 , and overestimates the observed Dst by a factor of 2 on day 177,1982 . Thus as good as the predictor is, there is room for improvement over (1) and (2). The next section explores whether the discrepant variability is systematic.

\section{Storm Time Dst Prediction From $A E_{w}$ and $\mathrm{AL}_{w}$}

The correlation coefficients derived in Figures 3a and 4a for each storm separately are typically better than the ones obtained from the annual prediction of Dst based on (1) and (2). For each of the 21 storms a separate fit was determined after the manner of (1) and (2). In some cases the most disturbed $D s t$ section was found to be better represented by a straight line fit of $D s t$ to $A E_{w}$ or $A L_{w}$. This procedure generated 21 pairs of functions describing a $D s t$ based on $A E_{w}$ and a further 21 pairs for $D s t$ based on $A L_{w}$. All of these functions are shown in Figure 9 with Dst $\left(A E_{w}\right)$ in Figure 9a and Dst $\left(A L_{w}\right)$ in Figure $9 b$. Equations (1) and (2) are shown in Figure 9 as the thick lines. Several features of this figure shed light on the preceding section. First, the storm with the largest $A E_{w}$ and $A L_{w}$ (storm 11) is quite dissimilar from the other 20 storms, hence we will exclude it for the following analysis. Second, low $A E_{w}$ segment of all the lines typified by (1) pass through a common point; for $A E_{w}$ this is $D s t=0$ at $A E_{w}=150 \mathrm{nT}$, while for $A L_{w}$ it is $D s t=0$ at $A L_{w}=-60 \mathrm{nT}$. The slopes range from -0.1 to -0.6 for Dst $\left(A E_{w}\right)$ and from 0.125 to 0.5 for Dst $\left(A L_{w}\right)$. This spread in slopes suggest that different physical conditions accompany different storms. Our present understanding of the responsible current systems is insufficient to determine the physical processes involved. Referring back to Figure 9, one notices that not every storm has a distinct second component to its prediction line. When a second component is present, this leads to a steepening of the Dst $\left(A_{w}\right)$ relationship. In about half of the cases the second part of the curve is an exponential, which leads to a continuously steepening relationship with increasing $l A_{w} l$.

This diversity of functional forms for the 21 storms accounts for the fact that fitting to a short period (e.g., the few days associated with a storm) can lead to very good correlation coefficients for individual events, whereas application of a fixed prediction formula (equations (1) and (2)) over longer periods (e.g., a year) leads to poorer correlation coefficients.

\section{Discussion}

The results of this study are complementary to earlier work and extend it in several ways. For weak geomagnetic activity our results agree with Akasofu [1981b], in that there is a linear correlation between the Dst and $A E$ in his case and $A_{w}$ in ours. However, our results clearly demonstrate that the "constant" of proportionality is not a universal constant but varies dramatically from one time interval to another; see Figure 9. There seems to be dependence on some other aspect of the magnetosphere-ionosphere system (perhaps a conductivity, a preconditioning of the magnetosphere, etc.) that needs to be identified. Cade [1993] verified that this additional dependence is not a simple solar cycle or seasonal one. This unknown dependence must conceal the primary reason why IMF- $D$ st $-A$ indices correlations (as reviewed by Feldstein [1992]) never find a unique prediction function. Indeed, the work of Pisarsky et al. [1989] encounters this same problem in 


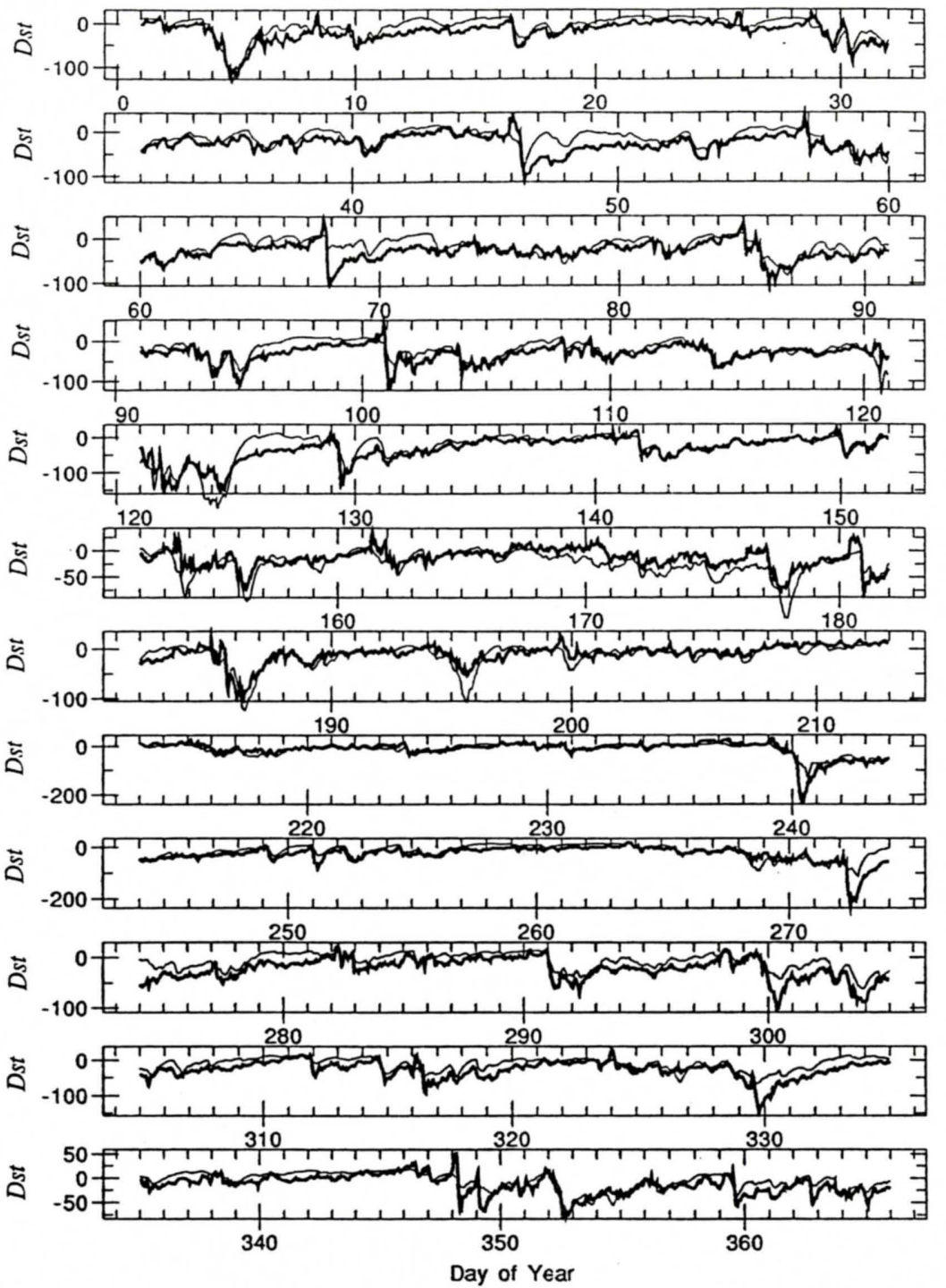

Figure 7. Dst (bold curve) and Dst $\left(A E_{w}\right)$ for 1982. Notice that the Dst scale is different for each month.

a different way. In their Dst (IMF) they develop a functional form which depends on the peak $D s t$ value for the interval being modeled. Our conclusion in this context is that the unknown relationship is more important than noise in the correlation due to poor statistics or the use of proxy indices to represent the physical process (auroral currents and ring currents).

Our second major finding is that during disturbed conditions the ring current and the driven component of the auroral current are strongly correlated. This correlation shows that the ring current builds up over a period of time and in our formulation the $D s t$ is related to the weighted sum of the $A$ indices over the recent past. For our specific weighting function (see the appendix) an attenuation time of 9.4 hours characterizes the weighting. Again, this result is consistent with earlier work [Davis and Parthasarathy, 1967; Kamide and Fukushima, 1971]. It goes further and shows that for each storm a high correlation coefficient is obtained when Dst and $A_{w}$ indices are compared (Figures 3 and 4 ) but that the specific relationship varies from one storm to the next (Figure 9). Since the optimal weighting parameter is $w=0.9,(\mathrm{~A} 1)$ in the appendix implies that the current hourly $A$ index value contributes only $10 \%$ to the prediction for Dst . The dependence on past history thus extends well beyond the 9.4 hours, the characteristic time

Table 2. Correlation Coefficients, Observed Dst Versus Predicted $D s t$ as a Function of $A E_{w}$ and $A L_{w s}$, for the Years 1978-1968

Correlation Coefficients

\begin{tabular}{lll}
\cline { 2 - 3 } Year & Dst $\left(A E_{w}\right)$ & Dst $\left(A L_{w}\right)$ \\
\hline 1978 & 0.7645 & 0.8012 \\
1979 & 0.7032 & 0.7654 \\
1980 & 0.7113 & 0.7920 \\
1981 & 0.7722 & 0.8180 \\
1982 & 0.7753 & 0.7927 \\
1983 & 0.7224 & 0.7755 \\
1984 & 0.8081 & 0.8242 \\
1985 & 0.7525 & 0.7898 \\
1986 & 0.7453 & 0.6050 \\
\hline
\end{tabular}




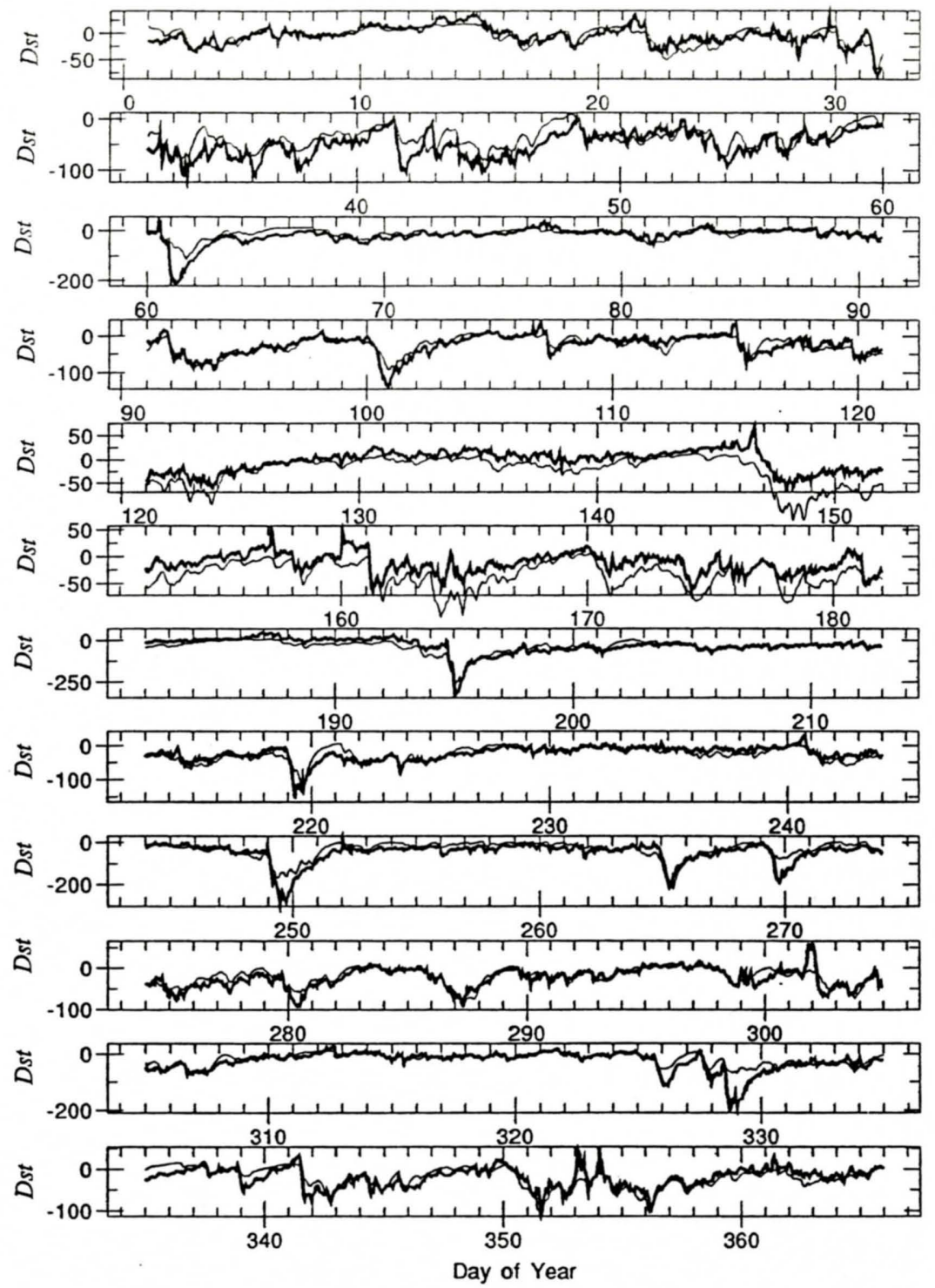

Figure 8. Dst (bold curve) and Dst $\left(A E_{w}\right)$ for 1978. Notice that the Dst scale is different for each month.

of the weighting function since 9.4 hours represents only a single $e$-folding of parametric influence. An even longer dependence on past history was found by Söraas and Davis [1968] when correlating energetic electron intensities at synchronous altitude with $K_{p}$. They found that a sum of $K_{p}$ over the past 10 days was necessary to obtain a geomagnetic correlation with these electron intensities. Koons and Gorney [1991] found a similar result when developing a neural network model of the relativistic electron flux at geosynchronous orbit driven by the planetary magnetic index $K_{p}$.

Given an unknown control of the coupling between auroral currents and ring currents at both low and high levels of activity, it is difficult to establish physical insight into the functional relationship between these currents and specifically among the indices acting as proxy for them. However, two separate functional relationships appear to be justified: a linear dependence for low geomagnetic activity and either a steeper proportionality or an exponential relationship under storm time conditions. The linear response at low activity, also found by Akasofu [1981b], would clearly argue that both current systems have a strong directly driven component under these conditions. This inference is based on the earlier assumption that the 1-hour $A$ indices are predominantly responsive to directly driven processes. Under quiet conditions the time rates of change of both the $A$ and $D s t$ indices is slow compared to the time constant used in constructing the $A_{w}$ indices. This explains why Akasofu [1981b] using just $A E$ and we (using $A E_{w}$ ) both obtain good linear correlation with $D s t$. However, the unknown parameter controlling the range of different slopes in Figure 9a makes it extremely difficult to determine the relative importance of directly driven processes (represented by $A E$ ) and cumulative processes (represented by $A E_{w}$ ).

Under strong geomagnetic activity the resolution of this issue is unambiguous (see Figures 2, 3, and 4): The past history of the directly driven component is accumulated to build up the ring current $(D s t)$. In Figure 9 the storm analysis even indicates that, as the magnitude of the $A_{w}$ increases, Dst increases exponentially (or at least faster than the linear relationship characteristic of quiet intervals would indicate). 

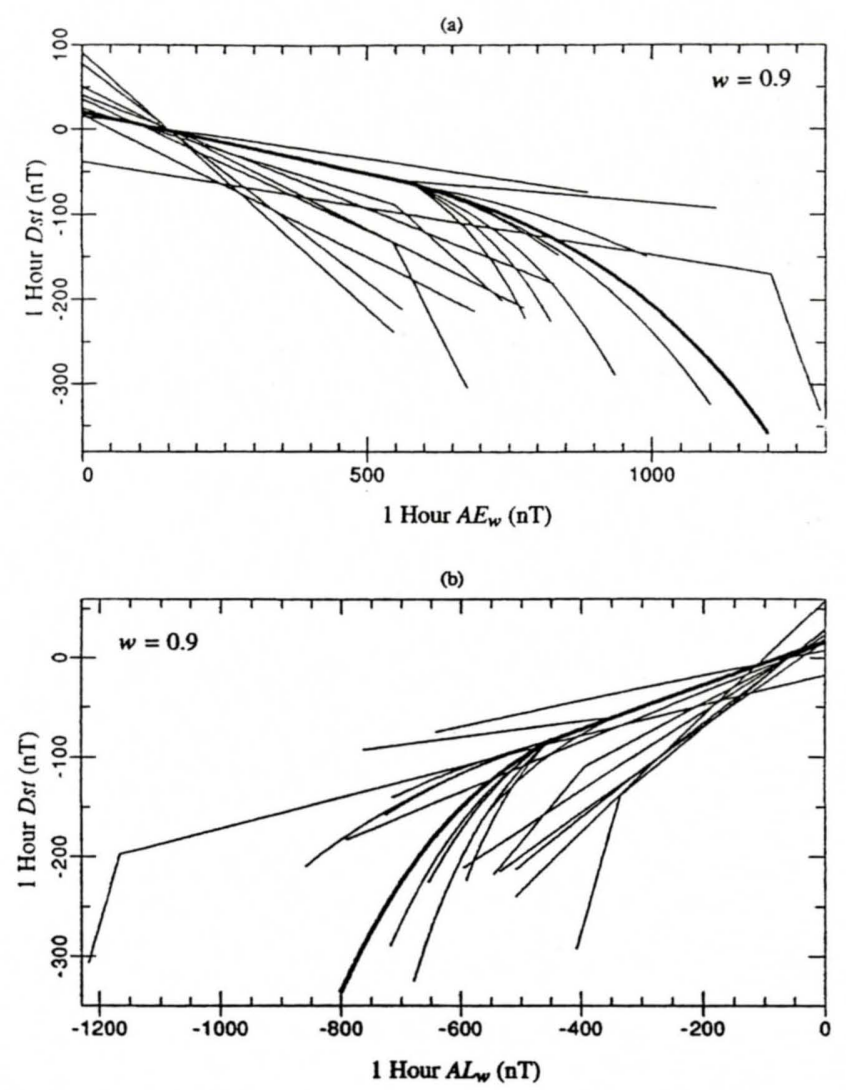

Figure 9. Dependence of Dst on (a) $A E_{w}$ and (b) $A L_{w}$. The relationships given by (1) and (2) are thick and the relationships for individual storms 1-21 are thin lines.

This result would be consistent with that inferred by Lanzerotti et al. [1978], who showed that fluctuations associated with magnetospheric transport increase exponentially with $K_{p}$. A similar conclusion was reached by Mozer [1971].

A final comment on procedure used to predict $D s t$ from the $A$ indices is in order. The hourly $A$ indices are assumed to represent a directly driven process, hence they are readily shut down by changes in the IMF. For this reason they should not show a long decay time. In contrast, the ring current builds up over many hours but decays over several days (see Figure 1). Our weighting algorithm (see appendix) has only one time constant, yet very good correlation between $A_{w}$ and $D s t$ is obtained through all phases of a storm. This is contrary to the simple consideration of what should happen during the recovery phase of a large storm. As the "driving force" is reduced or even goes away, auroral electrojets should show only a nondriven "unloading" response unrelated to ring current decay. However, the correlation we have obtained would suggest a strong coupling to the auroral current system even in the second and third days of the recovery phase.

\section{Conclusions}

The conclusions that can be drawn from this study are summarized as follows:

1. Both the hourly $A E$ and $A L$ indices, when weighted and summed over the recent past, generate new indices $A E_{w}$ and $A L_{w}$ that correlate very well with the $D s t$ index. The weighting function that describes the recent past has a time constant of
9.4 hours for both indices. For 20 of the 21 largest storms during solar cycle 21 , this procedure leads to correlation coefficients greater than 0.8 .

2. There is a linear relationship between the $A E_{w}$ (or $A L_{w}$ ) and $D s t$ during intervals of low $A E_{w}$.

3. During disturbed (storm time) intervals of large $A E_{w}$, the relationship between $D s t$ and $A E_{w}$ (or $A L_{w}$ ) can be either linear or exponential. In examples for which it is linear, the slope is steeper than for quiet intervals (compare conclusion 2).

4. Under both quiet and disturbed conditions, the function relating Dst to $A E_{w}$ (or $A L_{w}$ ) varies from one time interval to the next. This suggests that an additional controlling parameter needs to be identified and included in the correlation. During nonstorm conditions, this hypothesized additional parameter can cause the slope of the linear relationship to vary by factors of 4 to 5 .

Conclusion 4 implies that no unique functional relationship exists simultaneously for $A E$ (IMF) and Dst (IMF). However, by using the indices $A E_{w}$ or $A L_{w}$ defined by weighted averages of $A E$ and $A L$ over the recent past, the Dst can be predicted with correlation coefficients better than 0.75 under all conditions. From an applications standpoint, the $A$ indices are always available in close to real time. The present work shows that a real time forecast of $D s t$ is thus possible. Moreover, because the prediction of Dst entails an integration of such available parameters over the "recent past," the method offers a potential for a few hours' forecast capability. Work is ongoing in this area, as well as on the search for an additional physical parameter that seems to control the Dst response functions (compare conclusion 4).

\section{Appendix}

The auroral 1-hour indices, $A E, A L, A U$, and $A O$ are considered primarily as "directly driven," in that impulse current components occur on faster timescales and hence do not make a significant contribution to these hourly auroral indices. The directly driven convection electric field is also responsible for build up of the ring current and consequently the ring current index Dst. However, the ring current takes many hours to build up and so is better viewed as a "summation" of directly driven activity over the recent past. Unfortunately, the explicit form of the appropriate summation is unknown. Davis and Parthasarathy [1967] summed the 10 prior hourly $A E$ values to produce a parameter that varied with Dst. Kamide and Fukushima [1971] scaled the prior $A E$ values by an exponential weighting function and then added these scaled values to produce a parameter that represented the $D s t$ variation. More recently, Wrenn [1989] compared the 3-hour $A_{p}$ index to $D s t$ by summing preceding $A_{p}$ values with a weighting factor that diminished toward earlier times. Both Kamide and Fukushima [1971] and Wrenn [1989] apply a weighting that decreases with time into the past. Thus both methods can be characterized by an effective time constant. In this work we have followed the procedure described by Wrenn [1987] to develop a summed weighted index $A E_{w}(t)$ from the hourly $A E(t)$ :

$$
A E_{w}(t)=(1-w) A E(t)+w A E_{w}(t-1)
$$

The weighting parameter $w$ is selected so as to specify the degree of significance given to past $A E$ values. A value of $w=$ 0 would make the new index identical to $A E(t)$, whereas a value 
of 1 would generate an index totally independent of the present value of $A E(t)$. In this nomenclature $t$ is the time in hours, and so $A E_{w}(t-1)$ refers to the $A E_{w}$ value 1 hour earlier. Equation (A1) can also be expressed as a summation of all earlier $A E(t)$ values:

$$
A E_{w}(t)=(1-w) \sum_{n=0}^{\alpha} w^{n} A E(t-n)
$$

This form makes it readily evident that older values of $A E$ receive progressively smaller weighting (i.e., the $w^{n}$ factor decreases with $n$ since $0<w<1$ ). This weighting term in (A2) has an effective time constant.

$$
\tau=\frac{1 \text { hour }}{\ell n(1 / w)}
$$

Values of $\tau$ range from less than an hour for $w<1 / e=0.368$ (which is not particularly relevant to our study) to $\tau$ values greater than 1 hour for $w>0.368$, that is, $\tau=1.96,9.5$, and 24.5 hours, respectively, for $w=0.6,0.9$, and 0.96 . The time constant $\tau$ specifies the "lag" time by which the weighting factor $w^{n}$ in (A2) has decreased by a factor of $e$.

In this study we have applied (A2) to the entire time series of $A E(t), A L(t), A U(t)$, and $A O(t)$ from 1978 to 1986 so as to construct new time series called $A E_{w}(t), A L_{w}(t), A U_{w}(t)$, and $A O_{w}(t)$ for a specified value of $w$. For this study the optimal effective time constants $\tau$ were found to be less than 24 hours. Thus the error introduced in (A2) by not going back to times prior to 1978 is negligible except for the first few days in 1978.

Acknowledgments. This research was supported in part by NASA grant NAG5-1484 and NSF grant ATM-89-13230 to Utah State University. One of us, W. B. Cade III, was supported through the Air Force Institute of Technology. This study made use of the CEDAR Data Base at NCAR, which is supported by the National Science Foundation. The authors are grateful to one of the referees whose suggestions considerably helped clarify the presentation of this research.

The Editor thanks L. R. Lyons and another referee for their assistance in evaluating this paper.

\section{References}

Akasofu, S. I., Prediction of development of geomagnetic storms using the solar wind-magnetosphere energy coupling function $\varepsilon$, Planet. Space Sci., 29, 1151, 1981a.

Akasofu, S. I., Relationships between the $A E$ and $D s t$ indices during geomagnetic storms, J. Geophys. Res., 86, 4820, 1981 b.

Burton, R. K., R. L. McPherron, and C. T. Russell, An empirical relationship between interplanetary conditions and Dst, J. Geophys. Res., 80, 4204, 1975.
Cade, W. B., A correlative comparison of geomagnetic storms and auroral substorms using geomagnetic indices, M.S. thesis, Utah State Univ., Logan, 1993.

Davis, T. N., and R. Parthasarathy, The relationship between polar magnetic activity $D p$ and growth of the geomagnetic ring current, $J$. Geophys. Res., 72, 5825, 1967.

Davis, T. N., and M. Sugiura, Auroral electrojet activity index $A E$ and its universal time variations, J. Geophys. Res., 71, 785, 1966.

Fairfield, D. H., Advances in magnetospheric storm and substorm research: 1989-1991, J. Geophys. Res., 97, 10865, 1992.

Feldstein, Y. I., Modeling of the magnetic field of magnetospheric ring current as a function of interplanetary medium parameters, Space Sci. Rev., 59, 85, 1992.

Kamide, Y., and N. Fukushima, Analysis of magnetic storms with $D R$ indices for equatorial ring current field, Rep. Ionos. Space Res. Jpn., $25,125,1971$.

Kertz, W., Ring current variations during the IGY, Ann. Int. Geophys. Year, 35, 49, 1964.

Koons, H. C., and D. J. Gorney, A neural network model of the relativistic electron flux at geosynchronous orbit, J. Geophys. Res., 96, 5549, 1991.

Lanzerotti, L. J., D. C. Webb, and C. W. Arthur, Geomagnetic field fluctuations at synchronous orbit, 2., Radial diffusion, J. Geophys. Res., 83, 3866, 1978.

Mayaud, P. N. (Ed.), Derivation, Meaning, and Use of Geomagnetic Indices, Geophys. Monogr. Ser., Vol. 22, pp. 96-129, AGU, Washington, D. C., 1980.

Mozer, F. S., Power spectra of the magnetospheric electric field, $J$. Geophys. Res., 76, 3651, 1971.

Pisarsky, V. Y., Y. I. Feldstein, N. M. Rudneva, and A. Prigancova, Ring current and interplanetary medium parameters, Stud. Geophys. Geod., 33, 61, 1989.

Siscoe, G. L., Energy coupling between region 1 and 2 Birkeland current systems, J. Geophys. Res., 87, 5124, 1982.

Söraas, F., and L. R. Davis, Temporal variations of the $100 \mathrm{keV}$ to 1700 $\mathrm{keV}$ trapped protons observed on Satellite Explorer 26 during the first half of 1965, Rep. X-612-68-328, NASA Goddard Space Flight Center, Greenbelt, MD., 1968.

Sugiura, M., Hourly values of equatorial Dst for the IGY, Ann. Int. Geophys. Year, 35, 9, 1964.

Wrenn, G. L., Time-weighted accumulations $a p(\tau)$ and $K p(\tau), J$. Geophys. Res., 92, 10125, 1987.

Wrenn, G. L., Persistence of the ring current, 1958-1984, Geophys. Res. Lett., 16, 891, 1989.

W. B. Cade III, Air Force Space Command, Peterson AFB, CO 80914. J. J. Sojka and L. Zhu, Center for Atmospheric and Space Sciences, Utah State University, Logan, UT 84322-4405, (e-mail: fasojka@sojka. cass.usu.edu)

(Received September 2, 1993; revised August 31, 1994; accepted September 2, 1994.) 